\title{
Challenges in Treating Statin-Associated Necrotizing Myopathy
}

\author{
Patrick Webster $\mathbb{D}^{1},{ }^{1}$ Nicholas Wiemer $\mathbb{D}^{1},{ }^{1}$ Abdalhamid Al Harash $\mathbb{D},{ }^{2}$ Cody Marshall $\mathbb{D}{ }^{3}$ \\ Nazia Khatoon $(\mathbb{D})^{3}$ and Michael Lucke $\mathbb{D D}^{2}$
}

${ }^{1}$ Department of Internal Medicine, Allegheny Health Network, Pittsburgh, USA

${ }^{2}$ Department of Rheumatology, Allegheny Health Network, Pittsburgh, USA

${ }^{3}$ Department of Pathology, Allegheny Health Network, Pittsburgh, USA

Correspondence should be addressed to Nicholas Wiemer; nickwiemer@yahoo.com

Received 29 July 2020; Revised 29 January 2021; Accepted 12 February 2021; Published 24 February 2021

Academic Editor: Mario Salazar-Paramo

Copyright (c) 2021 Patrick Webster et al. This is an open access article distributed under the Creative Commons Attribution License, which permits unrestricted use, distribution, and reproduction in any medium, provided the original work is properly cited.

\begin{abstract}
Myalgia and mild elevation in muscle enzymes are common side effects of statin therapy. While these symptoms are generally selflimited, in rare cases, statin use is associated with an immune-mediated necrotizing myopathy caused by development of autoantibodies against HMG-CoA reductase. The primary presenting symptom of this condition is progressive symmetric proximal weakness that does not abate or worsens even after cessation of statin therapy and is associated with markedly elevated creatine kinase (CK) levels. To date, no randomized controlled trials have been conducted to identify the most effective treatment for statin-associated autoimmune myopathy. Treatment recommendations involve a combination of steroids and immunosuppressive drugs. This single-center case series highlights the clinicopathologic features diagnostic for statin-associated autoimmune myopathy as well as treatment challenges for the patient population. The series highlights a range of potential presentations, from mildly symptomatic despite highly elevated CK, to severe muscle weakness including dysphagia. Multiple patients required several immunosuppressant medications as well as intravenous immunoglobulin (IVIG) to achieve disease control. In this case series, marked improvement was noted in several diabetic patients with IVIG.
\end{abstract}

\section{Introduction}

Statin therapy has been shown to reduce the incidence of cardiovascular disease and is used routinely throughout medical practice. A common side effect of statin use is myalgia and mild elevation in muscle enzymes. These symptoms can result in muscle damage, but this reaction is self-limiting and abates with cessation of statin [1]. However, in rare cases, statin use can result in statin-associated autoimmune myopathy caused by the development of autoantibodies against HMG-CoA reductase [2]. Findings of a positive anti-HMG-CoA reductase (anti-HMGCR) antibody in the appropriate clinical context are highly suggestive of the diagnosis, which is further established with muscle biopsy pathology demonstrating a necrotizing myopathy with muscle-cell necrosis and regeneration [3]. Patients usually present clinically with progressive symmetric proximal weakness that may persist or worsen even after statin therapy is discontinued [2-6]. To date, no randomized controlled trials have been conducted to identify the most effective treatment for statin-associated autoimmune myopathy, but first-line treatment is typically a combination of steroids and immunosuppressive drugs $[4,5,7]$. In addition to immunosuppressive treatment, compelling data highlights the treatment benefits of IVIG $[4,6,8]$.

We report a single-center case series of seven patients with statin-associated autoimmune myopathy, highlighting the clinical heterogeneity in this disease as well as variable and outcomes associated with treatment via steroids, immunosuppression, and IVIG.

\section{Case Description 1}

A 71-year-old female with hypertension and hyperlipidemia treated with atorvastatin $40 \mathrm{mg}$ presented with 6 months of progressive proximal muscle weakness and myalgia in the 
upper and lower extremities with a creatine kinase (CK) level of $13,871 \mathrm{IU} / \mathrm{L}$. Of note, 2 years prior to this presentation, she experienced myopathy with elevated CK levels of 7,609 IU/L. ANA, anti Jo-1, SSA, SSB, and RNP were negative. The patient's atorvastatin was discontinued.

Electromyography (EMG) showed abnormal insertional activity and positive fibrillation waves consistent with a myopathic pattern. Muscle biopsy revealed myonecrosis without an inflammatory infiltrate (Figure 1). The antiHMG-CoA reductase (anti-HMGCR) antibody level was $>200 \mathrm{U} / \mathrm{ml}$.

The patient was initiated on prednisone $60 \mathrm{mg} /$ day. Proximal weakness improved, and CK decreased to 6,366 IU/L. Despite medication adherence, her symptoms recurred 6 weeks later, at which time CK rose to 12,000 IU/L, prompting treatment with $1 \mathrm{~g}$ of intravenous methylprednisolone daily for 3 days. She was started on azathioprine $150 \mathrm{mg} /$ day with a prednisone taper. The CK level downtrended to $311 \mathrm{IU} / \mathrm{L}$ over 10 months. The patient saw resolution of symptoms with 5/5 muscle strength in all 4 extremities after 9 months of treatment.

\section{Case Description 2}

A 54-year-old female presented with progressive muscle weakness over 2 months with impaired ambulation and multiple falls. She had a medical history of diabetes, hypertension, and hyperlipidemia treated with atorvastatin $80 \mathrm{mg}$. Labs were significant for a CK level of 24,300 IU/L. MRI of the left thigh showed extensive intramuscular edema and enhancement throughout the musculature (Figure 2). Muscle biopsy showed necrotizing myopathy with areas of degenerating and regenerating muscle fibers (Figure 3). Anti-HMGCR antibody was positive with a level of $>200 \mathrm{U} /$ ml. ESR, CRP, anti Jo-1, SSA, SSB, and RNP were within normal limits. Atorvastatin was changed to niacin, and the patient was started on prednisone $60 \mathrm{mg}$. The CK level decreased to 6,819 IU/L. Mycophenolate mofetil (MMF) $1000 \mathrm{mg}$ twice daily was added, with minimal symptomatic relief following 1 month of therapy. IVIG $2 \mathrm{~g} / \mathrm{kg}$ monthly was given for 1 year. Following 4 cycles of IVIG, the CK level continued to decrease to $1,610 \mathrm{IU} / \mathrm{L}$ and the patient's symptoms resolved. She was successfully tapered off steroids after a total of 5 months.

\section{Case Description 3}

A 64-year-old female with diabetes, hypertension, and hyperlipidemia presented with persistent lower extremity pain and weakness over 12 months. 6 months prior to presentation, atorvastatin was replaced with simvastatin due to suspicion of statin-associated myalgia.

Labs were significant for a CK level of 14,000 IU/L. ANA, SSA, SSB, and anti Jo-1 were negative. EMG showed increased insertional activity with positive fibrillation waves in the proximal upper and lower extremities and thoracic paraspinal muscles. Muscle biopsy showed necrosis with features of degeneration and regeneration without inflammation. Anti-HMGCR antibody was elevated at $167 \mathrm{U} / \mathrm{ml}$.
Prednisone and MTX were started, and due to persistent symptoms over 4 months, azathioprine $100 \mathrm{mg}$ /day was added. Over the following 8 months, weakness progressed and myalgias persisted, prompting a change to rituximab $1 \mathrm{~g}$, 2 weeks apart. Following the rituximab infusions, she was maintained on MMF $2 \mathrm{~g}$ and achieved remission over 4 years. Symptoms then flared with a CK level of 1,800 IU/L. Despite retreatment with rituximab $1 \mathrm{~g}$, CK remained $>1000 \mathrm{IU} / \mathrm{L}$ and proximal muscle weakness worsened. She was started on IVIG $2 \mathrm{~g} / \mathrm{kg}$ every 4 weeks while continuing $2 \mathrm{~g}$ MMF twice daily. She experienced symptomatic improvement, and CK dropped to $600 \mathrm{IU} / \mathrm{L}$. She subsequently achieved remission on IVIG $1 \mathrm{~g} / \mathrm{kg}$ every 4 weeks and MMF $2 \mathrm{~g}$ twice daily.

\section{Case Description 4}

A 76-year-old male with a past medical history of heart failure, coronary artery disease, hyperlipidemia, and diabetes presented with 6 weeks of bilateral upper and lower proximal muscle weakness. 2 years prior, he was started on $80 \mathrm{mg}$ atorvastatin. A CK level of 6,333 IU/L prompted discontinuation of atorvastatin. Over the next 4 weeks, symptoms progressed including development of dysphagia. EMG showed abnormal insertional activity in the form of positive waves and fibrillation potentials. Muscle biopsy showed nonspecific degenerating and regenerating muscle fibers without inflammation. Anti-HMGCR antibody was elevated to $131 \mathrm{U} /$ ml. ANA, anti-Smith, anti-dsDNA, ANCA, SSA, SSB, and RNP were negative. The patient was started on prednisone, MTX, and IVIG $2 \mathrm{~g} / \mathrm{kg}$ monthly. The patient's dysphagia resolved, ambulation improved, and CK normalized.

The patient was started on evolocumab for hyperlipidemia. He maintained on MTX $25 \mathrm{mg} /$ week and IVIG $2 \mathrm{~g} / \mathrm{kg}$ per month with a prednisone taper. IVIG was stopped with clinical improvement; however, the patient flared after withdrawal of this therapy. IVIG was restarted at $2 \mathrm{~g} / \mathrm{kg}$ a month, and azathioprine was started. With ongoing symptoms, azathioprine was changed to MMF. While on treatment, he developed a large right exudative pleural effusion with near-complete collapse of the right lung. Further evaluation with PET/CT did not show active myositis or evidence of malignancy, and the patient was maintained on IVIG monotherapy.

\section{Case Description 5}

A 66-year-old male with hypertension and hyperlipidemia on atorvastatin $80 \mathrm{mg}$ for 2 years presented with proximal leg weakness over 10 months with $4 / 5$ strength on examination. The CK level was 8,790 IU/L. Atorvastatin was discontinued, but symptoms worsened. On physical exam, the patient had 4/5 strength in the proximal upper and lower extremities. EMG showed fibrillation potentials and increased recruitment in the upper and lower extremities with truncal involvement. A muscle biopsy showed rare nonspecific atrophic fibers with no inflammation or necrosis. The anti-HMGCR antibody level was $>200 \mathrm{U} / \mathrm{ml}$. ANA, anticentromere, SSA, and SSB were negative. The patient 


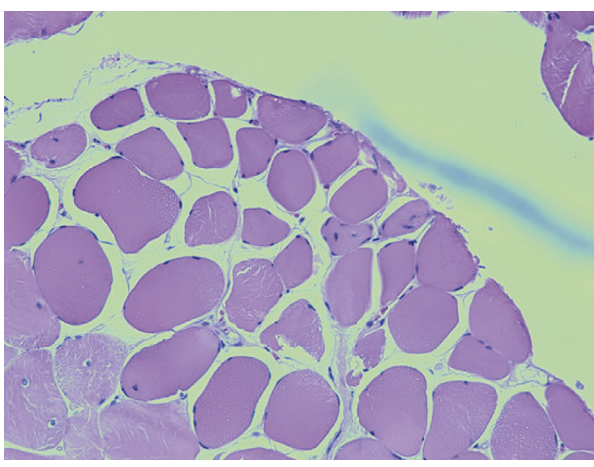

(a)

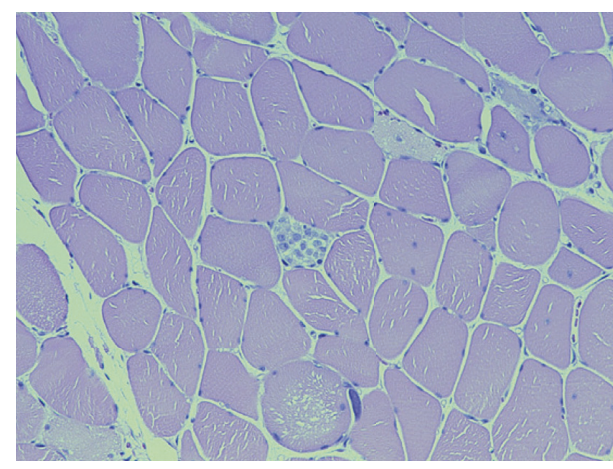

(b)

FIgURE 1: Regenerating (a) and degenerating (b) muscle fibers at $20 x$ magnification.

declined immunosuppression therapy and, despite persistent CK elevations ranging from 2,500 to $5,000 \mathrm{IU} / \mathrm{L}$, continued to have only mild weakness without interruption in daily activities.

\section{Case Description 6}

A 69-year-old male with a past medical history of antiphospholipid syndrome, diabetes, hypertension, and hyperlipidemia, on atorvastatin $20 \mathrm{mg}$ for four years, was hospitalized with acute hypoxic respiratory failure. The patient was found to have 3/5 hip flexor strength and reported having trouble walking for 3 months prior to admission. The patient had stopped atorvastatin 2 months prior to presentation. CK was 9,770 IU/L. EMG demonstrated a sensory and motor peripheral neuropathy. MRI showed increased T2 signal of the iliopsoas, gluteal, adductor, quadriceps, and semimembranosus muscles bilaterally. Muscle biopsy revealed necrotizing myopathy. Anti-HMGCR antibody was $>200 \mathrm{U} / \mathrm{mL}$. ANA, ANCA, anti Jo-1, CCP, PM SCL, and Lyme titers were negative. He was also found to have silent aspiration on modified barium swallow (MBS). His oxygenation improved, and he was discharged on prednisone $40 \mathrm{mg} /$ day.

After discharge, the patient's weakness worsened and prednisone was increased to $80 \mathrm{mg} /$ day. He was started on azathioprine $200 \mathrm{mg} /$ day and IVIG $700 \mathrm{mg} / \mathrm{kg}$ administered daily for 3 days per month with marked clinical improvement but persistent CK elevation after 4 months. He was maintained on IVIG $2 \mathrm{~g} / \mathrm{kg}$ monthly with azathioprine $50 \mathrm{mg}$ daily.

\section{Case Description 7}

A 72-year-old male with a past medical history of diabetes, atrial fibrillation, hypertension, and hypercholesterolemia presented with $4 / 5$ proximal muscle weakness on exam and CK of 7,802 IU/L. He had been treated with atorvastatin for the last 8 years. Although he had difficulty rising from a seated position, he reported that through a self-directed strengthening regimen, his strength had improved by $50 \%$.

Muscle biopsy confirmed a necrotizing myositis. The antiHMGCR antibody level was $192 \mathrm{U} / \mathrm{mL}$. Mi-2, PL-7, PL-12, $\mathrm{Ku}, \mathrm{EJ}$, OJ, anti Jo-1, and SRP were negative. The patient declined immunosuppressive treatment and had persistent 4/ 5 strength in his lower extremities and improved but persistent CK elevation at 2,419 IU/L. He continued to decline treatment as he was feeling well with exercising regularly.

\section{Discussion}

Statin-associated autoimmune myopathy is estimated to occur in approximately $2-3$ per 100,000 patients [9]. The primary presenting symptom is symmetrical proximal weakness, with clinicopathologic features including a marked increase of CK with the presence of autoantibodies against HMG-CoA reductase. All the patients encountered in this series were referred to our center with these hallmark diagnostic features (Table 1). While the etiopathogenesis of statin-associated autoimmune myopathy remains obscure, Basharat et al. reported that anti-HMG-CoA reductase myopathy is more common in patients with type 2 diabetes [10]. 5/7 patients in this cohort presented with a prior diagnosis of diabetes.

To date, no randomized controlled trials have been conducted to identify the most effective treatment for statinassociated autoimmune myopathy. Clinical reports have shown that steroids and immunosuppressive agents can improve symptoms $[4,5,11]$. Frequently prescribed therapies include prednisone with azathioprine, methotrexate, or mycophenolate mofetil $[4,8,12]$. Moreover, nearly half of the patients described in the literature were treated with three medications, most commonly with glucocorticoids, IVIG, and an additional immunosuppressant [12]. Our patient outcomes support the need for early immunosuppression in most patients. Although IVIG is often reserved for patients who have not benefitted from either steroid or oral immunosuppressive therapy, several suggestive studies have shown that IVIG could be used as a first-line monotherapy in select patients, particularly those with type 2 diabetes $[3,6]$. Indeed, 4 of 5 patients with diabetes in our series achieved marked improvement after initiation of IVIG, suggesting a potential earlier role for this therapy in diabetic patients.

It remains unclear why certain patients with statin-associated autoimmune myopathy and anti-HMGCR antibodies see a spontaneous resolution of symptoms while the 


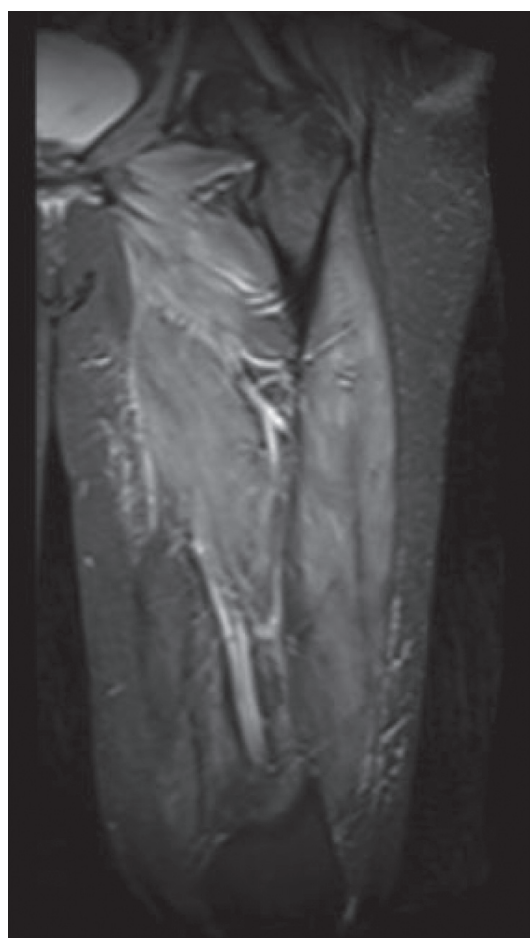

FIgURE 2: MRI and STIR weighted sequences of the left femur showing extensive intramuscular edema.

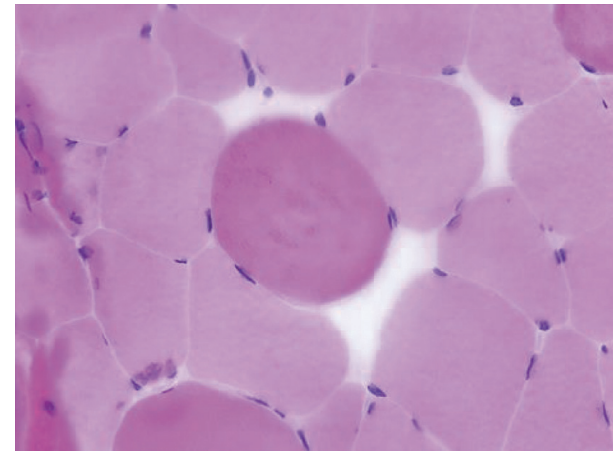

(a)

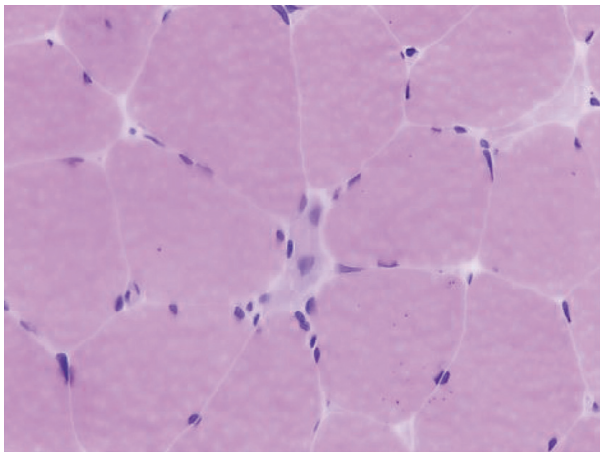

(b)

Figure 3: Degenerating (a) and regenerating (b) muscle fibers at $400 x$ magnification.

majority experience symptoms even after statin discontinuation. Patients 5 and 7 offer examples of this phenomenon. Both presented with mild cases of myopathy, and their symptoms and CK levels improved but remained elevated with statin cessation in the absence of immunosuppressive treatment. It has been postulated that although findings of muscle destruction persist as demonstrated by high $\mathrm{CK}$ levels, muscle regeneration may outpace destruction with resulting stability of symptoms [3]. Patient 7 implemented an early strengthening training, which in theory may assist with muscle regeneration.

Rechallenging with statin therapy may be considered in patients with mild myopathy or an isolated elevation in $\mathrm{CK}$, but alternative therapies are recommended in cases of statinassociated autoimmune myopathy. In this series, one patient was treated with the monoclonal antibody evolocumab without worsening of the disease. Another patient was treated with niacin successfully. 
TABLE 1: Summary of patients.

\begin{tabular}{|c|c|c|c|c|c|c|c|}
\hline Patient & $\begin{array}{l}\text { Age } \\
\text { (years) }\end{array}$ & Sex & $\begin{array}{l}\text { CK level } \\
(\mathrm{IU} / \mathrm{L})\end{array}$ & $\begin{array}{l}\text { Anti-HMGCR } \\
\text { antibody level } \\
(\mathrm{U} / \mathrm{mL})\end{array}$ & Primary biopsy findings & Statin & Therapy \\
\hline 1 & 71 & Female & 13,871 & $>200$ & $\begin{array}{l}\text { Degenerating and regenerating } \\
\text { muscle fibers without } \\
\text { inflammation }\end{array}$ & Atorvastatin & $\begin{array}{l}\text { Prednisone, } \\
\text { methylprednisolone, AZA }\end{array}$ \\
\hline 2 & 54 & Female & 24,300 & $>200$ & $\begin{array}{l}\text { Many regenerating and } \\
\text { occasional necrotic muscle fibers } \\
\text { with mild, patchy inflammation }\end{array}$ & Atorvastatin & Prednisone, MMF, IVIG \\
\hline 3 & 64 & Female & 14,000 & 167 & Necrotic muscle fibers & $\begin{array}{l}\text { Atorvastatin, } \\
\text { simvastatin }\end{array}$ & $\begin{array}{c}\text { Prednisone, MTX, AZA, } \\
\text { rituximab, MMF, IVIG }\end{array}$ \\
\hline 4 & 76 & Male & 6,333 & 131 & $\begin{array}{l}\text { Numerous regenerating, } \\
\text { degenerating, and atrophic } \\
\text { muscle fibers without } \\
\text { inflammation }\end{array}$ & Atorvastatin & $\begin{array}{c}\text { Prednisone, MTX, IVIG, } \\
\text { AZA, MMF }\end{array}$ \\
\hline 5 & 66 & Male & 8,790 & $>200$ & $\begin{array}{l}\text { Few, atrophic muscle fibers } \\
\text { Degenerating and regenerating }\end{array}$ & Atorvastatin & None \\
\hline 6 & 69 & Male & 9,770 & $>200$ & $\begin{array}{c}\text { muscle fibers without signs of } \\
\text { inflammation }\end{array}$ & Atorvastatin & Prednisone, AZA, IVIG \\
\hline 7 & 72 & Male & 7,802 & 192 & $\begin{array}{l}\text { Numerous degenerating and } \\
\text { regenerating fibers without } \\
\text { inflammation }\end{array}$ & Atorvastatin & None \\
\hline
\end{tabular}

HMGCR = HMG-CoA reductase, $\mathrm{AZA}=$ azathioprine, $\mathrm{IVIG}=$ intravenous immunoglobulin, $\mathrm{MMF}=$ mycophenolate mofetil, and $\mathrm{MTX}=$ methotrexate .

\section{Conclusion}

Statin-associated autoimmune myopathy is a rare complication of statin use that has potentially debilitating consequences. Treatment consists of prompt discontinuation of statins and early immunosuppression. In this case series, marked improvement was noted in several diabetic patients with IVIG. This finding supports the consideration of early initiation of IVIG in diabetic patients with highly active statin-associated autoimmune myopathy, potentially as a first-line agent.

\section{Data Availability}

The research data used to support the findings of this case series are included within the article.

\section{Conflicts of Interest}

The authors declare no conflicts of interest regarding publication of this article.

\section{References}

[1] B. A. Parker, J. A. Capizzi, A. S. Grimaldi et al., "Effect of statins on skeletal muscle function," Circulation, vol. 127, no. 1, pp. 96-103, 20131.

[2] Y. Allenbach, O. Benveniste, W. Stenzel, and O. Boyer, "Immune-mediated necrotizing myopathy: clinical features and pathogenesis," Nature Reviews Rheumatology, vol. 16, no. 12, pp. 689-701, 2020.

[3] A. Mammen, "Statin-associated autoimmune myopathy," New England Journal of Medicine, vol. 374, 2016.

[4] Y. Allenbach, A. L. Mammen, O. Benveniste et al., "Clinico-seropathological classification of immune-mediated necrotizing myopathies Zandvoort, the Netherlands," Neuromuscular Disorders, vol. 28, no. 1, pp. 87-99, 2018.

[5] S. Allenbach, S. Lohani, N. Tachamo, D. Poudel, and A. Donato, "Statin-associated autoimmune myopathy," JCR: Journal of Clinical Rheumatology, vol. 23, no. 3, pp. 149-154, 2017.

[6] C. Güngör and U. C. Wieshmann, "Severe statin-induced autoimmune myopathy successfully treated with intravenous immunoglobulin," BMJ Case Reports, vol. 13, no. 5, Article ID e234805, 2020.

[7] P. Grable-Esposito, H. D. Katzberg, S. A. Greenberg, J. Srinivasan, J. Katz, and A. A. Amato, "Immune-mediated necrotizing myopathy associated with statins," Muscle \& Nerve, vol. 41, no. 2, pp. 185-190, 2010.

[8] A. L. Mammen and E. Tiniakou, "Intravenous immune globulin for statin-triggered autoimmune myopathy," New England Journal of Medicine, vol. 373, no. 17, pp. 1680-1682, 2015.

[9] K. E. Hansen, J. P. Hildebrand, E. E. Ferguson, and J. H. Stein, "Outcomes in 45 patients with statin-associated myopathy," Archives of Internal Medicine, vol. 165, no. 22, pp. 2671-2676, 2005.

[10] P. Basharat, A. H. Lahouti, J. J. Paik et al., "Statin-induced anti-HMGCR-associated myopathy," Journal of the American College of Cardiology, vol. 68, no. 2, pp. 234-235, 2016.

[11] I. Albayda and A. L. Mammen, "Spectrum of immune-mediated necrotizing myopathies and their treatments," Current Opinion in Rheumatology, vol. 28, no. 6, pp. 619-624, 2016.

[12] I. Albayda and A. L. Mammen, "Spectrum of immune-mediated necrotizing myopathies and their treatments," Current Opinion in Rheumatology, vol. 28, no. 6, pp. 619-624, 2016. 